\title{
Calibration Method of an Evaluation System for the Body Conduction Sound Sensor
}

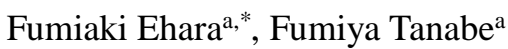 \\ ${ }^{a}$ National Institute of Technology, Ube College, 2-14-1 Tokiwadai, Ube-shi, Yamaguchi 755-8611, Japan \\ *Corresponding Author: ehara@ube-k.ac.jp
}

\begin{abstract}
In recent years, various household medical devices have been developed. Body conduction sound sensors that detect body sounds such as heart sounds, breath sounds and various sounds in the body are attracting attention because they are easy to measure. In this research, we aim to make an inexpensive evaluation system for evaluating body conduction sound sensors and to evaluate them. An evaluation speaker is made for the system. In order to calibrate the evaluation speaker, it is necessary to measure the amplitude of the vibration output from the speaker. The laser doppler vibrometer is used to measure it, and we calculated the frequency characteristic of the body conduction sound sensor using the measured results.
\end{abstract}

Keywords: body conduction sound senser, evaluation system, laser doppler vibrometer.

\section{Introduction}

In recent years, various household medical devices have been developed. Body conduction sound sensors that detect body sounds such as heart sounds, breath sounds and various sounds in the body are attracting attention because they are easy to measure ${ }^{(1-7)}$. Solitary death of elderly people is recognized as social problem in Japan. It is difficult to substantiate a system which monitor them using camera because of lack of privacy. There is a real need for a new monitoring system that conscious of privacy. The measurement becomes possible by using a body conduction sound sensor.

Evaluation of prototype body conduction sound sensor is necessary for development of it. To measure the frequency characteristic of the body conduction sound sensor, an evaluation method using a vibrator has been reported ${ }^{(8)}$.
However, the vibrator is very expensive, in this research, we aim to make an inexpensive evaluation system for evaluating body conduction sound sensors and to evaluate the sensor.

An evaluation speaker is made for the system. In order to calibrate the evaluation speaker, it is necessary to measure the amplitude of the vibration output from the speaker. The laser doppler vibrometer is used to measure it, and we calculated the frequency characteristic of the body conduction sound sensor using the measured results.

\section{Measurement System}

\subsection{The Body Conduction Sound Sensor}

The body conduction sound sensor is made by use of an improved small electret condenser microphone (Primo Co., EM189T). The microphone whose case is drilled a hole in and diaphragm is exposed is used. Figure 1 shows the container of the sensor that is made using polycarbonate as a material. Figure 2 is a picture of the sensor. The microphone is fixed in the center of the container, filled up with polyurethane resin. Then it can detect sounds inside of the body.

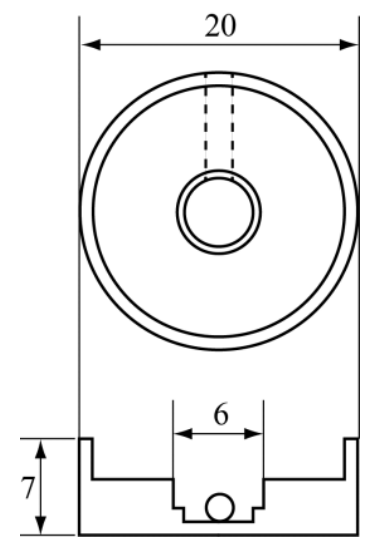

Fig. 1. Structure of the container of the sensor. 

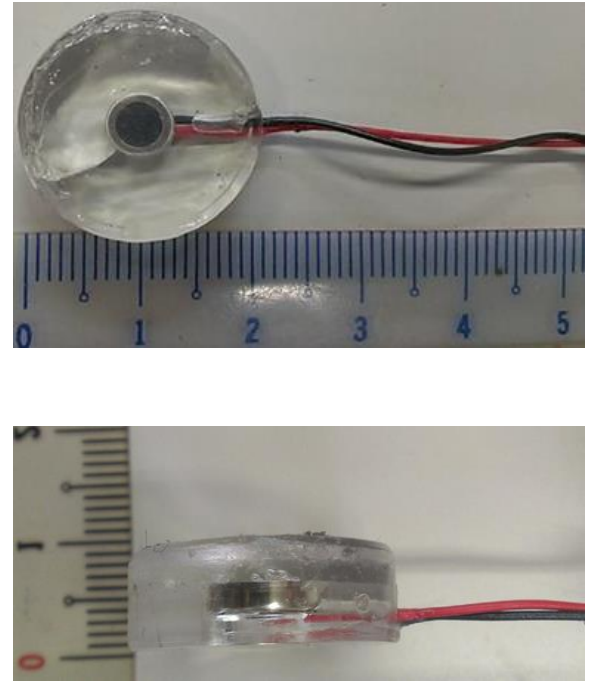

Fig. 2. The body conduction sound sensor.

\subsection{The Evaluation System}

Figure 3 shows the setup of the experiment to measure the frequency characteristic of the body conduction sound sensor. In the system, an evaluation speaker is used to evaluate the body conduction sound sensor. It is difficult to obtain the same waveform each time, and the reproducibility is low in the measurement of the body conduction sound by the actual human body. By using the evaluation speaker, it is possible to output the same waveform and measure with high reproducibility. The structure of the evaluation speaker is shown in Figure 4. A speaker (Tokyo Cone Paper MFG. Co., Ltd., F77G98-6) is installed in the center part of a transparent glass container and filled with polyurethane resin that is used also for the body conduction sound sensor. Figure 5 is a photograph of the produced evaluation speaker. There are two kinds of polyurethane resins, one with transparent color and one with white. In Fig. 5, a transparent one is used to make the structure easy to understand. In the experiment, we used a white one, because it was impossible to measure with a laser doppler vibrometer. (The laser doppler vibrometer will be explained in the next section.)

In the measurement, we place the body conduction sound sensor in close contact with the evaluation speaker. A sine wave signal is outputted from the computer and the vibration generated from the evaluation speaker is measured by the body conduction sound sensor. We measure the signal input to the evaluation speaker and the signal obtained from the body conduction sound sensor at the same time and measure the amplitude and the phase difference of the two signals. By changing the frequency of the sinusoidal wave generated from the speaker, we obtaine the frequency

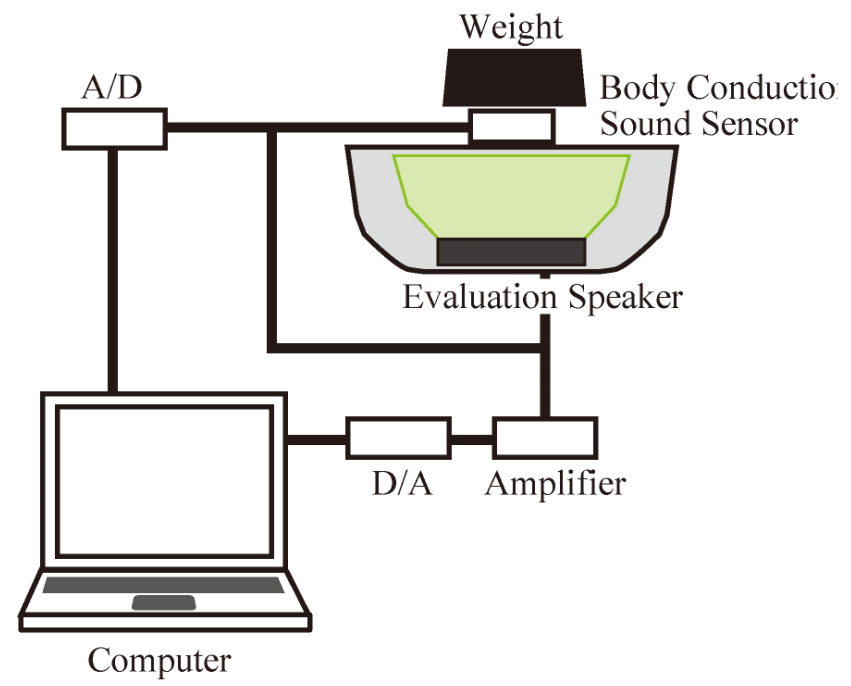

Fig. 3. The setup of the to measure the frequency characteristic of the body conduction sound sensor.

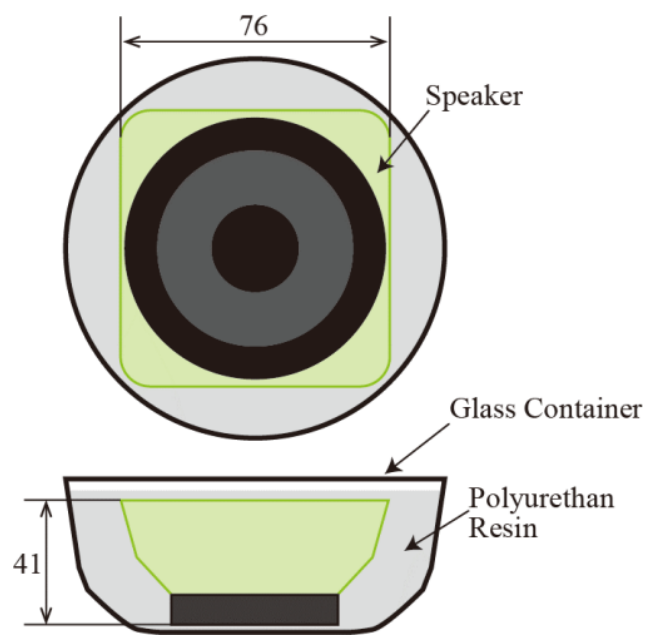

Fig. 4. The structure of the evaluation speaker.

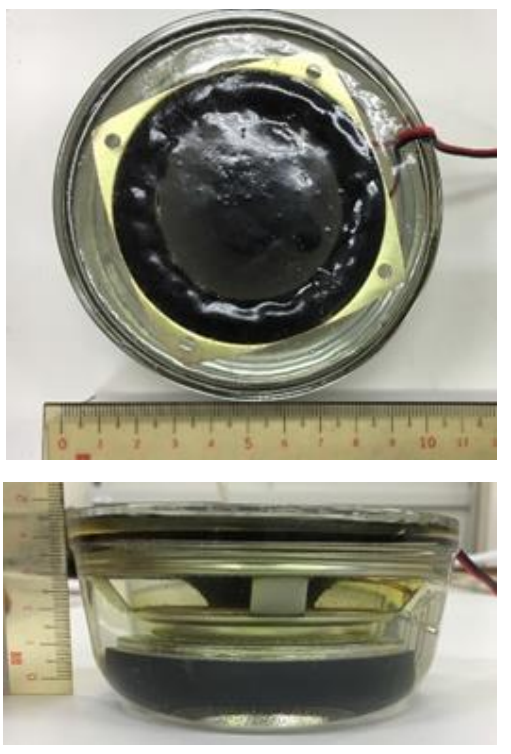

Fig. 5. The evaluation speaker. 
amplitude characteristic and the frequency phase characteristic of the body conduction sensor.

\subsection{Calibration of evaluation speaker}

For accurate measurement, calibration of the evaluation speaker is indispensable. In this study, a laser doppler vibrometer is used to measure the vibration generated from the evaluation speaker. Figure 6 shows the setup of the experiment to calibrate the evaluation speaker using a laser doppler vibrometer. Figure 7 is a photograph of the laser doppler vibrometer used in this study. We measure the signal input to the evaluation speaker and the signal obtained from obtained from the laser doppler vibrometer at the same time and measure the amplitude and the phase difference of the two signals. By changing the frequency of the sinusoidal wave generated from the speaker, we obtaine the frequency amplitude characteristic and the frequency phase characteristic of the evaluation speaker. By correcting the measurement result of the body conduction sound sensor using the measured frequency characteristic of the evaluation speaker, we can obtain the correct frequency characteristic of the sensor.

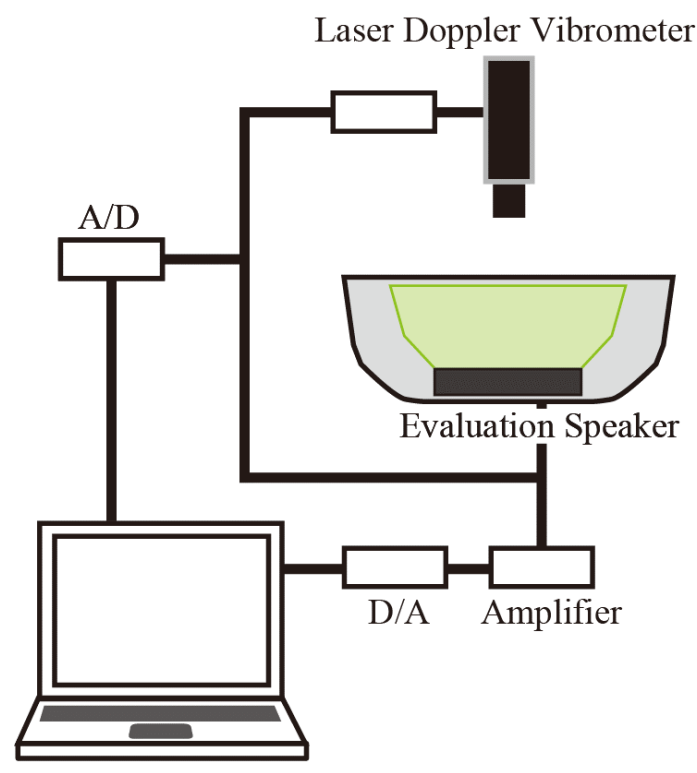

Computer

Fig. 6. The setup to calibrate the evaluation speaker using a laser doppler vibrometer.

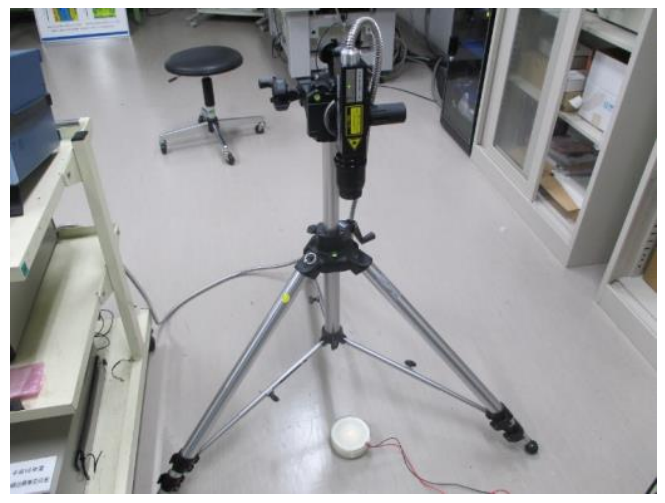

Fig. 7. The laser doppler vibrometer.

\section{Result}

We measure the frequency characteristic of the evaluation speaker using the laser doppler vibrometer. The frequency of the sine wave outputted from the computer is 100 to $10,000 \mathrm{~Hz}$, the sampling frequency is $50,000 \mathrm{~Hz}$, the number of data points is 131,072 points. The measurement

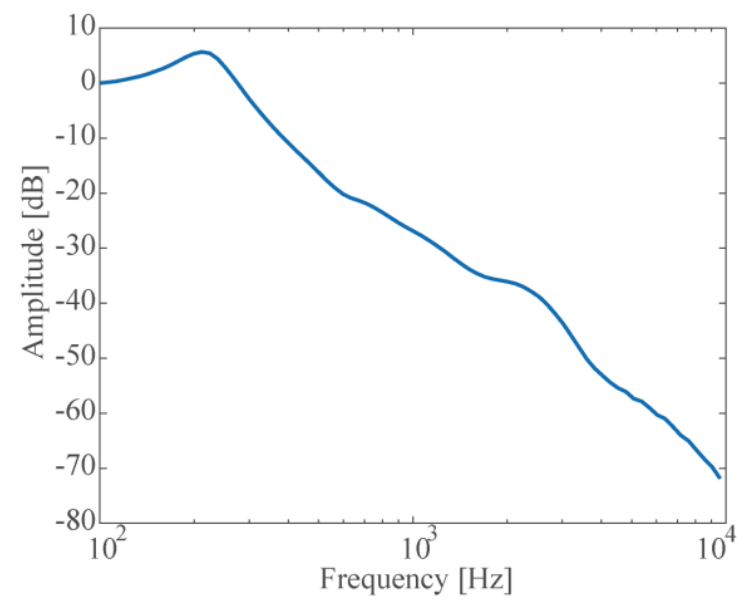

(a) Amplitude characteristic

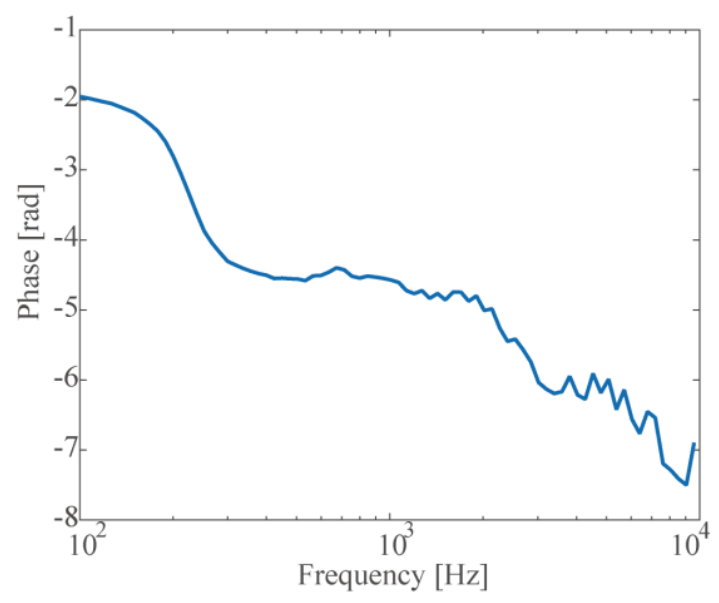

(b) Phase characteristic

Fig. 8. The frequency characteristic of the evaluation speaker. 
result is shown in the figure 8. (a) shows the frequency amplitude characteristic, and (b) shows the frequency phase characteristic. It is found that the amplitude characteristic of the evaluation speaker peaks around $200 \mathrm{~Hz}$, and the amplitude becomes smaller as the frequency becomes higher. Regarding the phase characteristics, it was confirmed that the phase lags as the frequency increases.

The frequency characteristic of the body conduction sound sensor is measured using the evaluation speaker. The measurement conditions are the same as the measurement of the evaluation speaker. The figure 9 shows the amplitude characteristic and the phase characteristic of the body conduction sound sensor. We can calibrate this measurement result using the result of the frequency characteristic of the evaluation speaker. The calibration results are shown in the figure 10. The amplitude characteristic of the body conduction sound sensor, it is confirmed that the amplitude increased with increasing frequency up to $10,000 \mathrm{~Hz}$.

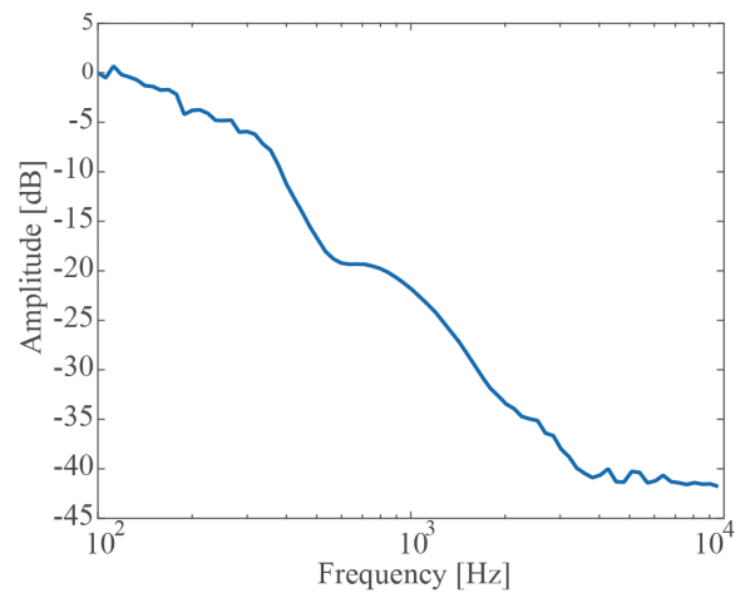

(a) Amplitude characteristic

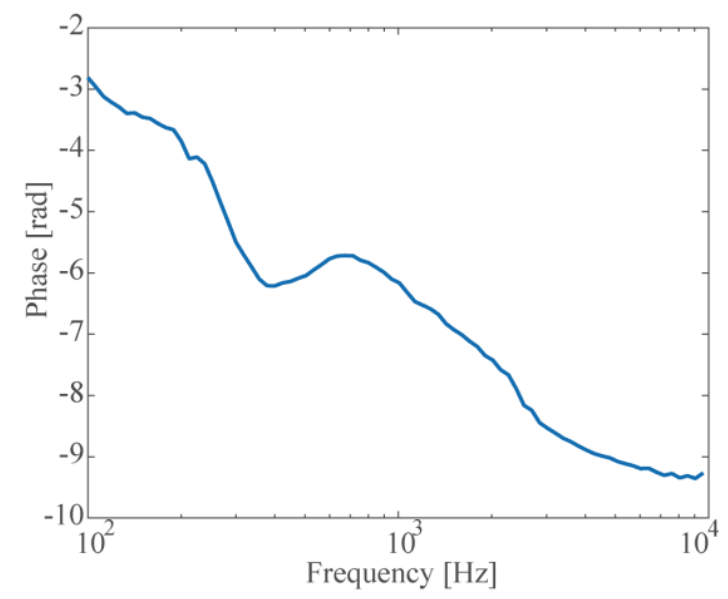

(b) Phase characteristic

Fig. 9. The frequency characteristic of the body conduction sound sensor.

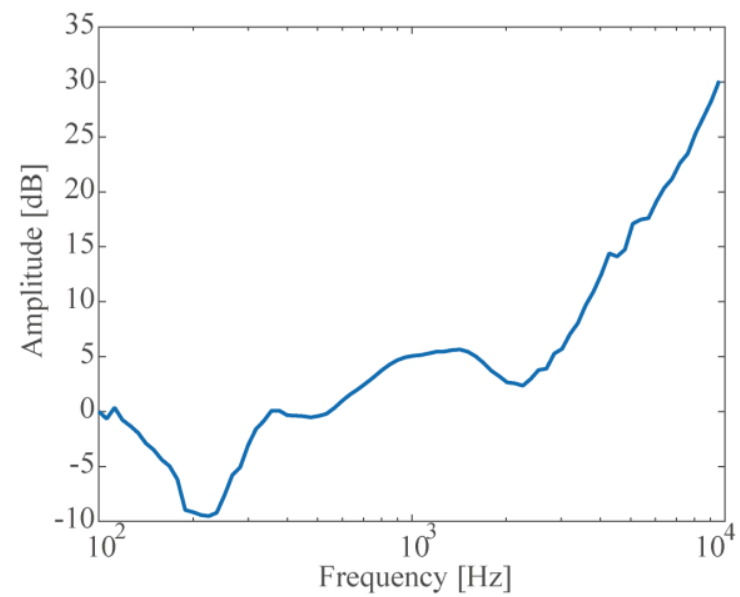

(a) Amplitude characteristic

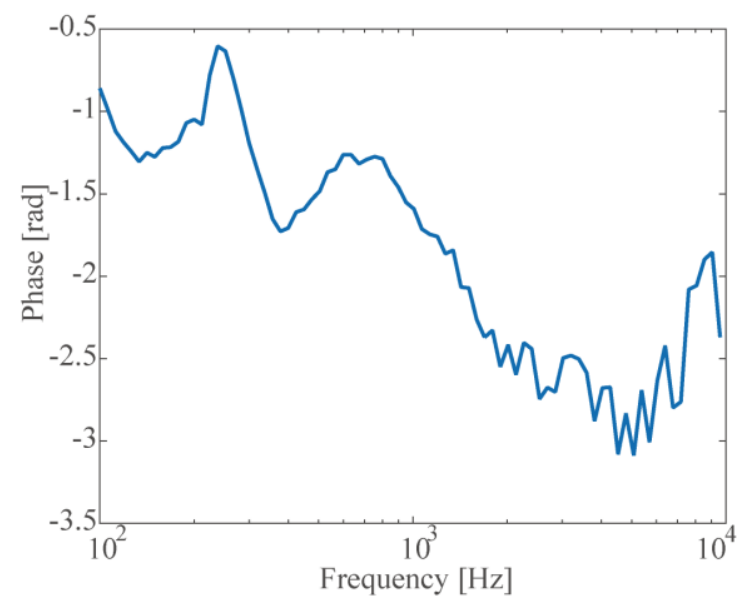

(b) Phase characteristic

Fig. 10. The frequency characteristic of the body conduction sound sensor after calibration.

\section{Conclusions}

In this paper, we made of body conduction sound sensor and evaluation speaker. We built an evaluation system to evaluate the body conduction sound sensor and measured the frequency characteristic of the body conduction sound sensor. To calibrate the evaluation speaker, a laser doppler vibrometer is used. we calculated the frequency characteristic of the body conduction sound sensor using the measured results.

The results of the measurement, the amplitude characteristic of the body conduction sound sensor, it is confirmed that the amplitude increased with increasing frequency up to $10,000 \mathrm{~Hz}$ and the phase is delayed as the frequency becomes higher.

In this study, we established an inexpensive system that can evaluate the body conduction sound sensor. 


\section{References}

(1) J.R. Bulgrin, B.J. Rubal, C.R. Thompson and J.M. Moody : "Comparison of Short-Time Fourier, Wavelet and Time-Domain Analysis of Intracardiac Sounds", Biomed Sci. Instrum. Vol.29, pp.465-472, 1993

(2) C.H. Wu, C.W. Lo and J.F. Wang : "Computer-Aided Analysis and Classification of Heart Sounds Based on Neural Networks and Time Analysis", Proc. IEEE Int. Conf. Acoust. Speech Signal Process, pp.355-3458, 1995

(3) Z. Jiang and S. Choi : "Development of Wireless Electronic Stethoscope System and Abnormal Cradiac Sound Analysis Method (Sound Characteristic Waveform Analysis)", The Japan Society of Mechanical Engineers, pp.140-147, 2005

(4) T. Hirahara and S. Shimizu : "Sensing various types sound generated inside the body using an ECM-based compact body-conducted sound sensor", Proc. Autumn Meet. Acoust. Soc. Jpn., 2009

(5) Fumiaki Ehara, Ryouhei Tanaka and Kanya Tanaka : "Development of a Wi-Fi-enabled Measurement System for a Body Conduction Sound", Journal of the Institute of Industrial Applications Engineers, Vol.3, No.4, pp.174-177, 2015

(6) Fumiaki Ehara, Takahiro Kubo and Kanya Tanaka : "Measurement System of Heart Rate Using a Mbed Microcontroller", INFORMATION, Vol.17 No.2, pp.545-550, 2014

(7) Fumiaki Ehara, Toshiaki Tsuruda and Kanya Tanaka : "Development of a Heart Rate Measurement Method Using Body Conduction Sound Sensor", Applied Mechanics and Materials, Vol.103, pp.616-621, 2012

(8) S. Shimizu, M. Otani and T. Hirahara : "Frequency characteristics of several non-audible murmur (NAM) microphones", Acoust. Sci. \& Tech., pp.139-142, 2009 